\title{
Low- and high-order gravitational harmonics of rigidly rotating Jupiter
}

\begin{abstract}
N. Nettelmann
Universität Rostock, Institut für Physik, 18051 Rostock, Germany

e-mail: nadine.nettelmann@uni-rostock.de

Received 11 July 2017 / Accepted 19 August 2017

ABSTRACT

The Juno Orbiter has provided improved estimates of the even gravitational harmonics $J_{2}$ to $J_{8}$ of Jupiter. To compute higher-order moments, new methods such as the concentric Maclaurin spheroids (CMS) method have been developed, which surpass the commonly used theory of figures $(\mathrm{ToF})$ method in accuracy. This progress raises the question whether ToF can still provide a useful service for deriving the internal structure of giant planets in the solar system. In this paper, I apply both the ToF and the CMS method to compare results for polytropic Jupiter and for the physical equation of state H/He-REOS.3-based models. An accuracy in the computed values of $J_{2}$ and $J_{4}$ of $0.1 \%$ is found to be sufficient in order to obtain the core mass safely within $0.5 M_{\oplus}$ numerical accuracy and the atmospheric metallicity within about 0.0004 . ToF to the fourth order provides that accuracy, while ToF to the third order does not for $J_{4}$. Furthermore, I find that the assumption of rigid rotation yields $J_{6}$ and $J_{8}$ values in agreement with the current Juno estimates, and that higher-order terms $\left(J_{10}\right.$ to $\left.J_{18}\right)$ deviate by about $10 \%$ from predictions by polytropic models. This work suggests that ToF 4 can still be applied to infer the deep internal structure of giant planets, and that the zonal winds on Jupiter reach less deep than $0.9 R_{\mathrm{J}}$.
\end{abstract}

Key words. planets and satellites: individual: Jupiter - planets and satellites: interiors

\section{Introduction}

The theory of figures (ToF; Zharkov \& Trubitsyn 1978) to third or fourth order, hereafter labeled respectively $\mathrm{ToF} 3$ and $\mathrm{ToF} 4$, is commonly used to compute the gravity field of the gas giant planets in the solar system (e.g., Saumon \& Guillot 2004; Helled 2011; Leconte \& Chabrier 2012; Nettelmann et al. 2012; Helled \& Guillot 2013; Miguel et al. 2016). While a theory to $n$th order allows for computation of the gravity field in terms of the gravitational harmonics up to $J_{2 n}$ only, Jupiter's gravity field before the current Juno mission was also measured up to $J_{6}$ only. Thus, third or fourth order theories seemed sufficient. Moreover, it is the low-order harmonics $J_{2}$ and $J_{4}$ that are particularly sensitive to the internal density distribution; they allow for derivation of the interior structure parameters core mass and envelope metallicity. Hence for a long time, the observational data of gravity field theories (e.g., ToF) and planet-interior parameters of interest formed a closed system.

This convenient situation has changed with the arrival of the Juno spacecraft at Jupiter. Juno's sensitivity limit makes it possible to measure the rigid-rotation contribution to the gravitational harmonics up to $J_{14}$ (Kaspi et al. 2010). High-order moments yield clues to the properties of the zonal winds as the flows influence the density distribution, which in turn is the source function of the gravitational potential. Differential rotation due to zonal flows is predicted to entirely dominate the $J_{2 n}$ for $n \geq 14$, while it is predicted to be within a factor of ten of the prediction for a rigidly rotating planet for $J_{8}-J_{12}$ (Hubbard 1999; Kaspi et al. 2010; Cao \& Stevenson 2017). Since the wind contribution $\Delta J_{2 n}^{\text {wind }}$ is obtained by subtracting the theoretical values for a rigidly rotating planet from the observed ones
$\left(J_{2 n}^{\text {obs }}=J_{2 n}^{\text {rigid }}+\Delta J_{2 n}^{\text {wind }}\right)$, it is also important to have accurate knowledge of the rigid-rotation contribution.

For that purpose, Hubbard (2013, hereafter H13), developed the concentric Maclaurin spheroids (CMS) method. This method yields demonstratively good agreement with the exact Bessel solution for an $n=1$ polytrope model of Jupiter. Deviations have been found to be about $5 \times 10^{-5}$ in $J_{2}$ to $2 \times 10^{-4}$ in $J_{20}$ (Wisdom \& Hubbard 2016), or to be of the order of $2 \times 10^{-3}$ (Cao \& Stevenson 2017). However, comparison of the exact Bessel solution to the ToF 3 results (H13; Wisdom 1996) has led to the conclusion that ToF 3 is insufficiently accurate for modeling Jupiter (Wisdom 1996). This raises the question of what accuracy in the low-order moments is desired for inferring Jupiter's internal density distribution, and which methods can provide that.

The classical view of a Jupiter-like gas giant is that of a well-defined core embedded into an $\mathrm{H} / \mathrm{He}$-rich envelope, in which case one can search for the mass of the core and the heavy element mass fraction of the envelope and use the measured planet radius and $J_{2}$ value to determine both (Militzer et al. 2008). However, recent gas giant formation models challenge that simple picture. Simultaneous accretion of gas and planetesimals might naturally lead to a gas-enriched, that is, diluted core (Venturini et al. 2016), where the metallicity is predicted to decrease outward as a result of the slow convective timescale compared to the accretion rate during formation (Helled \& Stevenson 2017); a diluted core may remain permanent due to the inhibition of convection once a compositional gradient has become established (Vazan et al. 2016). Diluted cores have been found to enhance the predicted atmospheric metallicity of Jupiter models (Fortney \& Nettelmann 2010). 

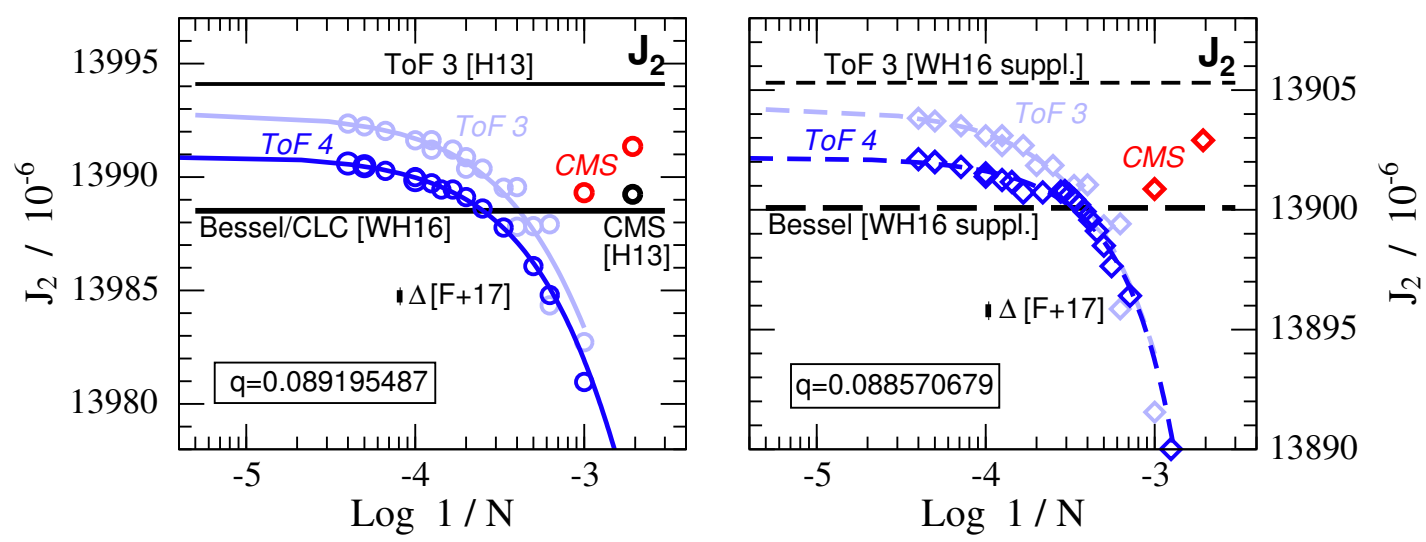

Fig. 1. Resulting $J_{2}$ values of polytropic models of rigidly rotating Jupiter for $q=0.089195487$ (left) as in H13 and Wisdom \& Hubbard (2016), and for $q=0.088570679$ (right) as in Wisdom (1996). Blue symbols: using ToF to fourth order, blue lines: respective fit curves, light blue: same as blue but using ToF 3, red symbols: using CMS method. Reference values are in black; black circle: CMS results of $\mathrm{H} 13$ for $N=512$, horizontal black lines: unknown value of $N$, in particular: thick black lines: Bessel/CLC results of Wisdom \& Hubbard (2016), thin black lines: ToF 3 results of H13 and Wisdom (1996). The vertical black arrow shows the current Juno uncertainty of $J_{2}$ (Folkner et al. 2017), here arbitrarily placed. The $x$-axis is number of radial grid points $N$.
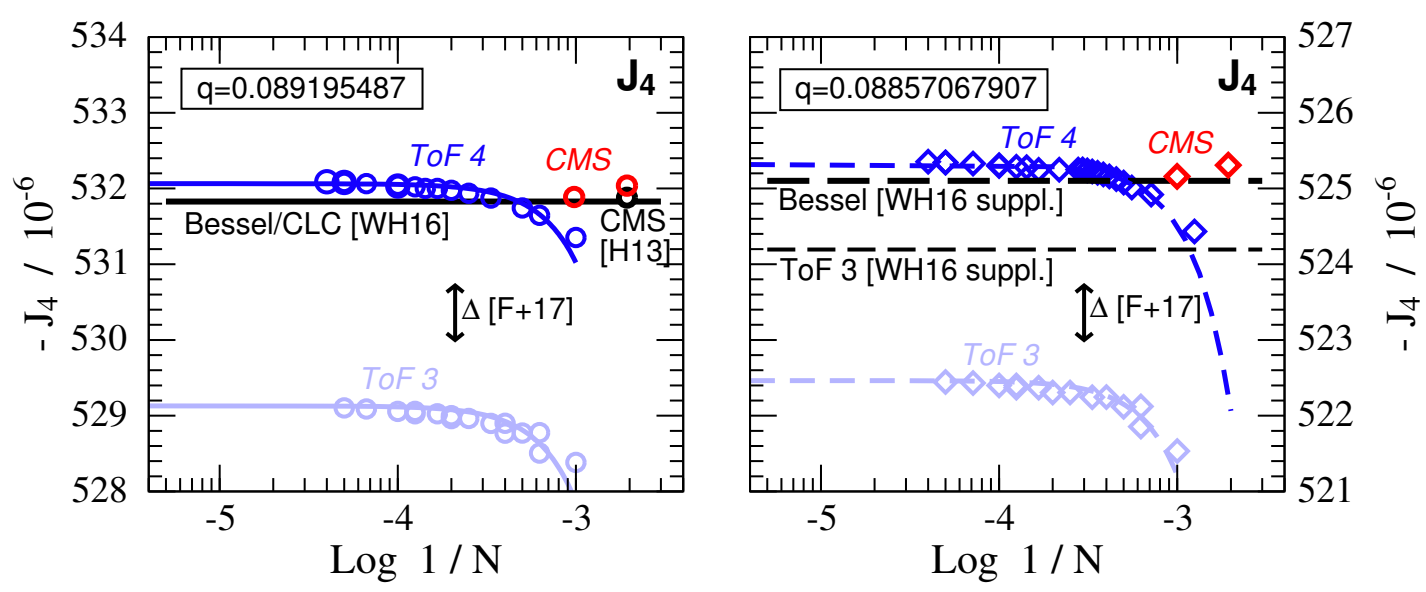

Fig. 2. Same as Fig. 1 but for $J_{4}$. The ToF 3 result of Hubbard (2013) exceeds the shown range of $J_{4}$ values.

In this paper, the uncertainty in the computed values for the low-order harmonics $J_{2}, J_{4}, J_{6}$ due to application of ToF 3 and ToF 4 is estimated by using the $n=1$ polytrope model (Sect. 2); the corresponding uncertainty in the derived core mass and envelope metallicity of Jupiter is estimated in Sect. 3. Finding this uncertainty to be small for ToF 4, I use this method in Sect. 4 to compute physical equations of state (EOS)-based Jupiter models that are designed to match the low-order harmonics $J_{2}$ and $J_{4}$ from Juno's first two low-periapse polar orbits around Jupiter (Folkner et al. 2017). Models are presented both for solid cores and for diluted cores, as well as for deep zonal wind corrections as proposed by Cao \& Stevenson (2017). For some of the Jupiter models, I compute the high-order moments using the CMS method (Sect. 5), thereby providing the first prediction of the high-order $J_{2 n}$ values for a model of an adiabatic, rigidly rotating Jupiter that matches the measured low-order moments. Conclusions are in Sect. 6. In Appendix A my implementation of the CMS method is validated for the linear density case, while in Appendix B the ToF coefficients are provided up to the fourth order.

\section{Polytropic models}

In this section, $n=1$ polytropic models are computed for $G M_{\mathrm{J}}=12.6686536 \times 10^{16} \mathrm{~m}^{3} / \mathrm{s}^{2}$, equatorial radius
$R_{\mathrm{eq}}=R_{\mathrm{J}}=71492 \mathrm{~km}$, and for two different rotation rates as represented by $q=0.0891954870$ (Wisdom \& Hubbard 2016) and $q=0.08857067907$ (Wisdom 1996), where $q=\omega^{2} R_{\mathrm{eq}}^{3} / G M$. I apply ToF to the third and fourth order as well as the CMS method. For both methods an iterative procedure is required to ensure the total mass is conserved, and that for the thus specified value of $K$ in the polytropic relation $P=K \rho^{2}$ hydrostatic balance holds.

With ToF I calculate the density at grid point $i$ using $\rho_{i}=$ $\sqrt{P_{i} / K}$, while with CMS method $\rho_{i}=\sqrt{0.5\left(P_{i}+P_{i+1}\right) / K}$ (H13), except for $i=0$ where $\rho_{0}=P_{0}=0$ in their respective units. As I find the dependence on the number of radial grid points, $N$, to be strong, I plot the resulting values of $J_{2}$ (Fig. 1), $J_{4}$ (Fig. 2), and $J_{6}$ (Fig. 3) against $N$.

Compared to the exact Bessel solution (Wisdom \& Hubbard 2016), the CMS method performs best and ToF 3 worst. In particular, ToF 3 underestimates $\left|J_{4} / 10^{-6}\right|$ by one to two times (depending on the implementation) the pre-Juno $1 \sigma$ error bar of $\sim 2$ (Miguel et al. 2016), and therefore predicts a higher atmospheric metallicity for Jupiter than ToF 4 does (Nettelmann et al. 2012).

$\mathrm{ToF} 4$, on the other hand, performs much better: the difference in $J_{4}$ to the exact Bessel solution amounts to only about $15 \%$ of the total pre-Juno error bar, and the differences in both $J_{4}$ and $J_{6}$ are still smaller than the uncertainties of the current Juno data. The influence of the error in $J_{4}$ due to the application 


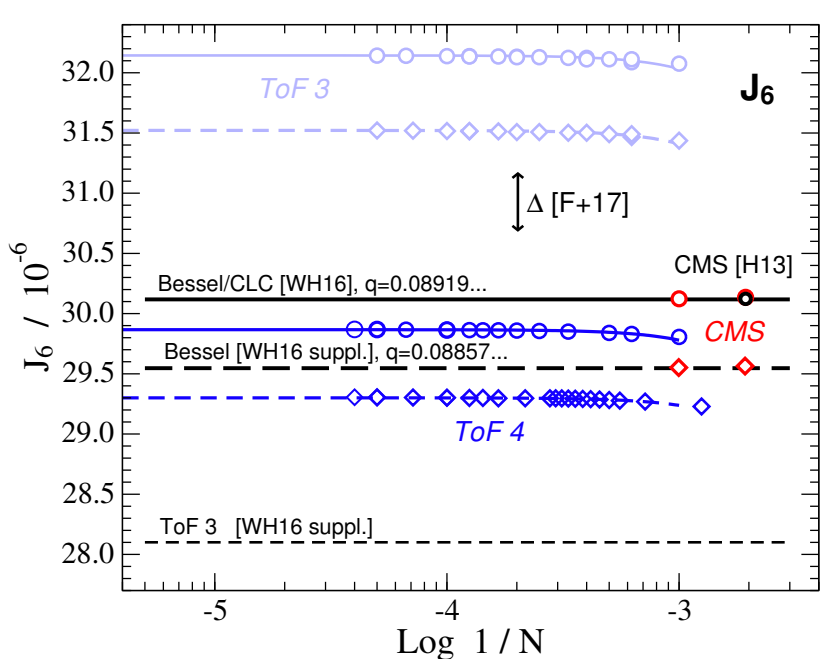

Fig. 3. Same as Figs. 1 and 2 but for $J_{6}$ and results for different $q$ values merged into a single panel, distinguished by respectively solid lines/circles and dashed lines/diamonds. The ToF 3 result of Hubbard (2013) exceeds the shown range of $J_{6}$ values.

of ToF 4 on the predicted envelope metallicity and core mass of Jupiter can be considered negligible. In the following section, I investigate whether this is also the case for $J_{2}$, the error bar of which is $5 \times$ the current Juno estimate of $2 \times 0.272 \times 10^{-6}$ (Fig. 1).

\section{Interior models and $\boldsymbol{J}_{\mathbf{2}}$}

The observed value of $J_{2}$ allows for insight into the internal structure of Jupiter, as different internal density distributions may yield different values of $J_{2}$ to be compared against the observed one. In this section I investigate how sensitive that dependence is. In particular, I consider the resulting uncertainty in the derived core mass $\left(M_{\text {core }}\right)$ and atmospheric metallicity $\left(Z_{\text {atm }}\right)$ due to the technical uncertainty in $J_{2}$, which results from applying ToF 4 to compute the gravitational harmonics. For this purpose, simple models are computed for which I assume a constant metallicity throughout Jupiter's envelope. Although further details of the procedure do not influence the resulting quantities we are interested in (the uncertainties), I give them for completeness: the envelope is separated into an outer, He-poor part of helium abundance $Y_{\text {atm }}=0.238$ in agreement with the Galileo entry probe value, and a He-rich inner envelope that accounts for the remaining helium to yield a total $\mathrm{He} / \mathrm{H}$ mass ratio of 0.275 in agreement with estimates for the protosolar cloud. The transition takes place at pressure $P_{\text {trans }}=8 \mathrm{Mbar}$. The envelope adiabat runs through the temperature-pressure point of $423 \mathrm{~K}$ at 22 bars as measured by the Galileo entry probe. At the outer boundary at one bar this yields $T_{1}=170 \mathrm{~K}$, which I adopt as the outer boundary condition for the Jupiter models. Figure 4 shows the resulting uncertainties in $M_{\text {core }}$ and $Z_{\text {atm }}$ as a function of the assumed value of $J_{2}$.

According to Fig. 4 , the error in $J_{2}$ of about $2 \times 10^{-4}$ due to applying ToF 4 maps onto an uncertainty of $0.02 M_{\oplus}$ in Jupiter's core mass and 0.0001 in $Z_{\text {atm }}$. Furthermore, an uncertainty of $0.1 \%$ in $J_{2}$ (twice the horizontal length of gray lines) would imply an uncertainty of $\sim 0.1 M_{\oplus}$ in core mass and 0.0004 in $Z_{\text {atm }}$. Thus, the uncertainties due to applying ToF 4 can be considered tiny compared to the accuracy in internal structure properties we are interested in, which is about $10 \%$ (e.g., $\sim 1 M_{\oplus}$ in core mass). Moreover, the uncertainty from this source of error is clearly smaller than the uncertainty due to the material input
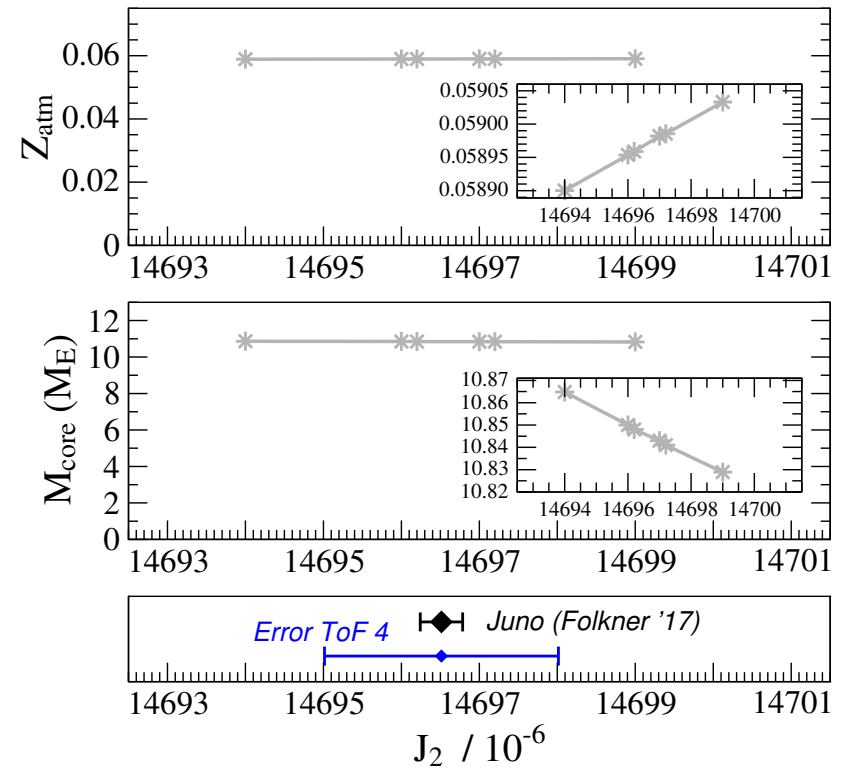

Fig. 4. Uncertainty in the derived values for core mass and atmospheric metallicity due to the assumed uncertainty in $J_{2}$ for interior models assuming constant envelope- $Z$. For $\Delta J_{2}$ about $10 \times$ the current Juno uncertainty, corresponding to $2 \times$ the estimated error from applying ToF 4 (bottom panel) the core mass uncertainty amounts to $\sim 0.04 M_{\oplus}$ (middle panel), while the uncertainty in $Z_{\mathrm{atm}}$ is found to be less than 0.0002 (upper panel). These uncertainties are small.

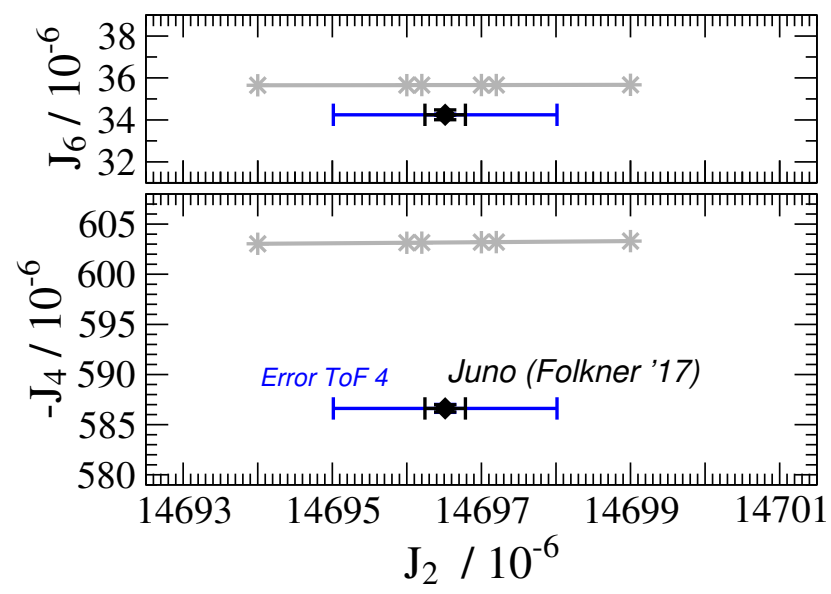

Fig. 5. Same as Fig. 4 but for $J_{4}$ (lower panel) and $J_{6}$ (upper panel).

physics like the EOS, which is at best of the order of $1 \%$. Furthermore, the additional error in $J_{4}$ and $J_{6}$ which results from the uncertainty in $J_{2}$ due to applying ToF 4 amounts to only $0.04 \%$ for $J_{4}$ and $0.03 \%$ for $J_{6}$ (Fig. 5). From Figs. 4 and 5 I therefore conclude that ToF 4 yields quantitatively useful density distributions for Jupiter.

\section{Results for Jupiter}

In this section I construct models that aim to match the tight current Juno constraints on $J_{2}$ and $J_{4}$ and are based on $\mathrm{H} / \mathrm{He}$ REOS.3 (Becker et al. 2014, hereafter B14). In Sect. 4.1 I assume rocky cores and rigid rotation, while in Sect. 4.2 I assume diluted cores or take into account the shift due to winds. 


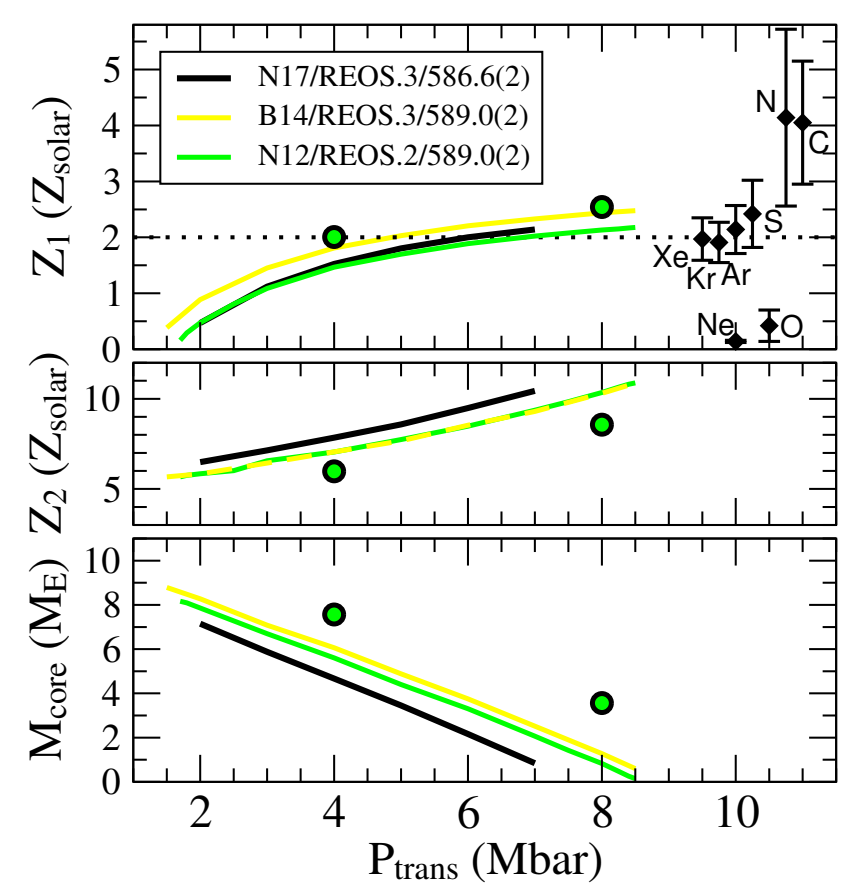

Fig. 6. ToF 4 based three-layer Jupiter models that are designed to match observed $J_{2}$ and $J_{4}$ values. This work: black solid, B14: yellow, N12: green. Measured atmospheric particle abundances of the elements as labeled are scaled by their protosolar particle abundance values (Lodders 2003). I use $Z_{\text {solar }}=0.015$.

\subsection{Models with solid cores and rigid rotation}

The models in this section are three-layer models and constructed as in Nettelmann et al. (2012), hereafter N12. The only but important difference to the models of Sect. 3 is that threelayer models allow for different heavy element abundances in the two envelopes, so that two free parameters $\left(Z_{1}=Z_{\text {atm }}\right.$ in the outer and $Z_{2}$ in the inner envelope) are available for adjusting the two low-order harmonics $J_{2}$ and $J_{4}$. If this can be achieved and if in addition $Z_{1} \geq 2 \times$ solar, consistent with the observed heavy noble gas abundances in Jupiter's atmosphere, I consider a model as acceptable for Jupiter.

Figure 6 shows these new models as a function of $P_{\text {trans }}$. They are similar to the ToF 4 based models of N12, who applied H-REOS.2 and He-REOS.1, and of B14 who applied H-REOS.3 and He-REOS.3 as in this work. The biggest differences are the narrower range in possible transition pressure and the lower $Z_{1}$ values compared to $\mathrm{B} 14$. The latter is mainly a direct consequence of reducing $\left|J_{4} / 10^{-6}\right|$ from 589 to 586.6. The lower $Z_{1}$ values also tend to reduce $J_{2}$, requiring more heavy elements in the deep interior to compensate for that. Slightly higher $Z_{2}$ values then leave less mass to build the core, so that finally a smaller set of models (a smaller range of $P_{\text {trans }}$ values for which $M_{\text {core }} \geq 0$ ) is found. In contrast, the difference between these models and the N12 results was mainly due to differences in the helium EOS at outer envelope pressures. These new results confirm that ab initio $\mathrm{H} / \mathrm{He}-\mathrm{EOSs}$ yield rather low atmospheric metallicities for Jupiter. Compared to $\lesssim 1 \times$ solar (Hubbard \& Militzer 2016; Wahl et al. 2017b), $\lesssim 2.5 \times$ solar (N12), $3 \times$ solar (B14), I here obtain $Z_{\text {atm }} \lesssim 2 \times$ solar, out of which acceptable models have $P_{\text {trans }}=6-7$ Mbar.

An inaccuracy in $J_{4}$ of about 2.4/600 (0.4\%) (compare black and yellow curves in Fig. 6) seems to induce a rather large uncertainty of $\Delta M_{\text {core }}=2 M_{\oplus}$ in core mass; but a $0.1 \%$ uncertainty in $J_{4}$ might still lead to $\Delta M_{\text {core }}=0.5 M_{\oplus}$ for three-layer models.

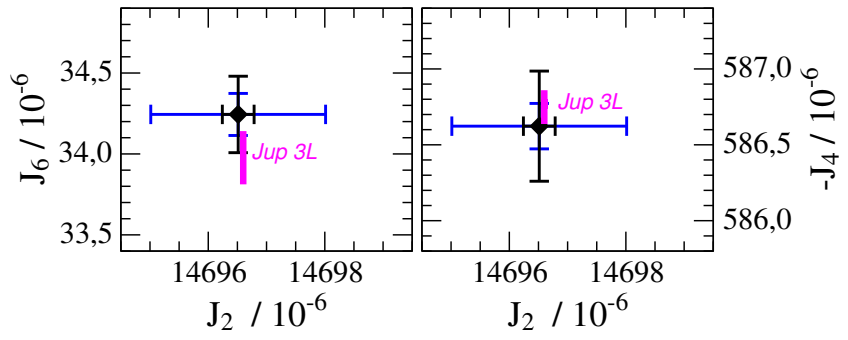

Fig. 7. (Left panel) Resulting ToF 4-based $J_{6}$ values of Jupiter models (magenta). The $J_{4}$ value of these models (magenta, right panel) was adjusted to match the Juno $J_{4}$ measurement. Black diamonds: Juno measurements, blue error bars: estimated uncertainty due to applying ToF 4 according to Figs. 1-3.

However, this estimate is probably a far upper bound as the models in B14 were computed with a smaller number of grid points of $N \sim 2000$ compared to $\sim 12000$ in this work.

Figure 7 compares the resulting $J_{6}$ value of the ToF 4-based Jupiter models that match the Juno $J_{2}$ and $J_{4}$ values to the Juno measurement of $J_{6}$. Models with $P_{\text {trans }}=4-7$ Mbar are within the observational uncertainty of $J_{6}$, while models with lower transition pressures are within $2 \sigma$ of the observational uncertainty. The computational error from ToF 4 is much smaller than that.

\subsection{Models with diluted cores or zonal winds}

$\mathrm{Ab}$ initio $\mathrm{H} / \mathrm{He}$ EOS-based Jupiter models with rock-ice cores and without zonal winds become notoriously low in atmospheric heavy element abundances. On the other hand, diluted cores have been found to enhance $Z_{\text {atm }}$ by up to $50 \%$ (Fortney \& Nettelmann 2010), while zonal winds directly affect the $J_{2}$ and $J_{4}$ values to be matched by rigidly rotating models (Militzer et al. 2008; Cao \& Stevenson 2017) and thus also influence the derived $Z_{\mathrm{atm}}$ value. While precise predictions on the dynamic contributions $\Delta J_{2 n}$ to the observed values depend on the differential rotation pattern and their mathematical description (Kaspi et al. 2010; Zhang et al. 2015; Cao \& Stevenson 2017), it is predicted that the effect on the low-order $J_{2 n}$ increases with the depth of the winds (Kaspi et al. 2010; Cao \& Stevenson 2017) and that the effect on the low-order $J_{2 n}$ is small and in the direction of reducing the absolute $J_{2 n}$ values (Hubbard 1999; Kaspi et al. 2010; Cao \& Stevenson 2017). Here I calculate Jupiter models as in Sect. 4.1 but by assuming a diluted core of rock mass fraction $Z_{3 \text {, Rocks }}=0.2$, the rest being inner mantle material, and by including zonal wind corrections as proposed by Cao \& Stevenson (2017) for half-amplitude width (HAWD) values of 0.8 and 0.9 . The latter quantity is defined as the distance to the rotation axis where the azimuthal wind velocity has weakened by a factor of two from its maximum value farther out.

As shown in Fig. 8, the zonal wind corrections lead to lower values in $Z_{1}$ and $M_{\text {core }}$. This is not a surprise, since the absolute values of $J_{2}$ and $J_{4}$ are reduced and thus demand a smaller mass density in the part of the planet planet where they are most sensitive, which is near $P \sim 1$ Mbar in the outer envelope. This behavior is in line with the observation of Militzer et al. (2008) who, in order to enhance the resulting envelope $Z$ value, suggest zonal wind effects on $J_{2}$ and $J_{4}$ in the opposite direction of what zonal wind models that fit the observed wind speeds predict.

For HAWD $=0.8 \mathrm{I}$ do not obtain any acceptable Jupiter model because the $\Delta J_{2 n}$ are too large: both $Z_{1}$ and $M_{\text {core }}$ would become negative. For HAWD $=0.9$, there is a restricted range 


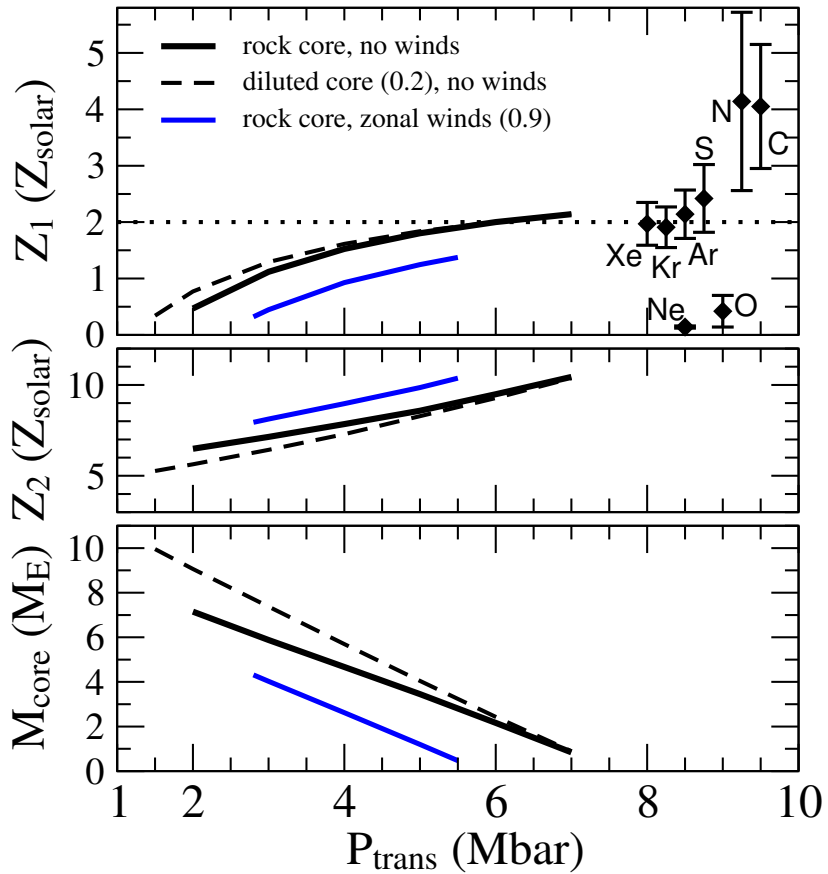

Fig. 8. Jupiter models with diluted cores of central rock mass fraction $Z_{3 \text {, Rocks }}=0.2$ (black dashed) or with zonal winds according to HAWD $=0.9$ (solid blue). The solid black curves are the same as in Fig. 6. I use $Z_{\text {solar }}=0.015$.

of solutions at $P_{\text {trans }}=3-5.5 \mathrm{Mbar}$, for which $Z_{1}$ barely reaches $1.5 \times$ solar. Adiabatic H/He-REOS.3-based Jupiter models thus suggest the vertical extent of the winds to be less than $0.9 R_{\mathrm{J}}$ $(\sim 7000 \mathrm{~km})$.

Assuming a diluted core and adiabatic envelopes, the $Z_{1}$ value can be lifted, but only to less than its maximum value obtained for core-less models. The enhancement in $Z_{1}$ can indeed reach up to $50 \%$ for the largest core mass found here, but then the base $Z_{1}$ value is small anyway. Therefore, as Fig. 8 shows, diluted cores do not significantly enhance $Z_{\mathrm{atm}}$ for $\mathrm{H} / \mathrm{He}-$ REOS-based models, but are helpful for larger core models (Wahl et al. 2017b) such as those obtained with the EOS of Militzer \& Hubbard (2013).

\section{High-order gravitational harmonics}

To compute the high-order gravitational harmonics of models that match the observed Juno values for $J_{2}$ and $J_{4}$, I use the density distributions of the models from Sect. 4 and apply the CMS method to them ${ }^{1}$. For that purpose, I convert the ToF-based density profile as a function of the mean radius of an equipotential surface to a density profile as a function of the equatorial radius of the same equipotential surface using the ToF 4-based figure functions. Then I reduce the number of radial grid points from $N \sim 12000$ to $N \sim 1000$ by assuming a radial spacing that decreases continuously from the middle to the boundaries. At layer boundaries, the jump in density is conserved as illustrated in Fig. 9.

\footnotetext{
1 One could of course use the CMS method right from the start; however, my current implementation of the CMS method runs orders of magnitudes slower than my implementation of the ToF method. Given the number of iterations necessary to fit both $J_{2}$ and $J_{4}$ according to the procedure outlined in Nettelmann (2011), I evaluated the accuracy gained as being not worth the enormous computational extra effort.
}

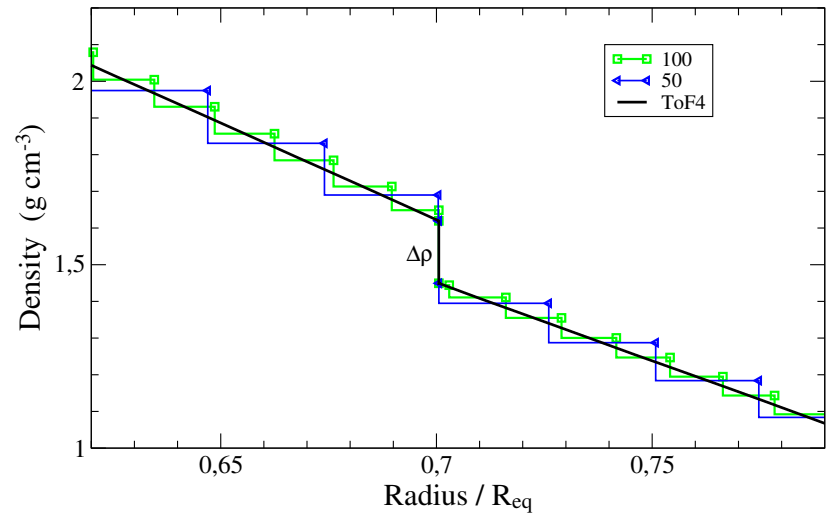

Fig. 9. Conversion of the ToF 4-based density profile (black) to a discrete grid as required by the CMS method, here illustrated for $N=50$ (blue) and $N=100$ (green).

Finally, Table 1 presents my results for the low- and highorder $J_{2 n}$ values of two models from Sect. 4.1, that is, for $P_{\text {trans }}=3$ Mbar (model J17-3a) and 6 Mbar (model J17-6a), and for one model that accounts for deep zonal winds through the corrections to $J_{2}$ and $J_{4}$ for HAWD $=0.9$ from Sect. 4.2 (model J17-4z). Resulting moments of order $\geq 6$ are not affected by the above described procedure within the number of digits given in Table 1 . This is shown by model variant $(b)$, where the $J_{2}$ value to be fitted was shifted by the difference ToF 4 minus CMS according to model variant $(a)$. The results are compared to the exact polytrope solution (Wisdom \& Hubbard 2016), to the DFT-MD-7.13 Jupiter model of Hubbard \& Militzer (2016), to the Juno measurements of Folkner et al. (2017), and to the same data but corrected for deep zonal winds as proposed by Cao \& Stevenson (2017).

Perhaps most interestingly, the resulting values for $J_{6}$ and $J_{8}$ for a rigidly rotating Jupiter are within the current Juno observational error bars. This may indicate that the winds are shallow. The $J_{8}$ value of model $\mathrm{J} 17-4 \mathrm{z}$ is also within the observational error bar and the reduction of its $\left|J_{8}\right|$ value by a few percent is much less than the few $10 \%$ estimate of Cao \& Stevenson (2017), whose $\Delta J_{8}$ estimate peaks for HAWD $=0.9$ compared to deeper (0.8) or shallower (0.975) depths. Furthermore, the highorder $J_{2 n}$ values of $n=1$ polytropic Jupiter differ by about $10 \%$ from the physical EOS-based Jupiter models. Thus it is important to provide the latter class of models as done in this work and in Hubbard \& Militzer (2016).

\section{Conclusions}

To infer Jupiter's internal density distribution, the relative accuracy in the computed values of $J_{2}$ and $J_{4}$ should be of the order of $0.1 \%$ (Figs. 4 and 6). According to $n=1$ polytropic models, ToF 4 can provide this accuracy, while ToF 3 can do so only for $J_{2}$ (Figs. 1 and 2). The error in $J_{2}\left(J_{4}, J_{6}\right)$ due to applying ToF 4, as measured by the difference to the exact solution, is about $5 \times$ $(1 / 2 \times, 1 / 3 \times)$ the current Juno uncertainties for these parameters. I conclude that these uncertainties are nevertheless sufficiently small for predicting Jupiter's internal density distribution. Other uncertainties, such as the thermal state, perhaps as a result of helium rain, may induce larger unknowns in our understanding of Jupiter (Nettelmann et al. 2015; Hubbard \& Militzer 2016; Mankovich et al. 2016; Wahl et al. 2017b).

The computed values of $J_{6}$ and $J_{8}$ of rigidly rotating Jupiter suggest that zonal winds are restricted to regions well above a 
Table 1. High-order moments of different models for rigidly rotating Jupiter.

\begin{tabular}{|c|c|c|c|c|c|c|c|c|}
\hline$J_{2 n}$ & $\begin{array}{l}\text { Juno } \\
{[\mathrm{F} 17]}\end{array}$ & $\begin{array}{c}\text { ToF-4 } \\
\text { J17-3a }\end{array}$ & $\begin{array}{c}\text { CMS-1000, } \\
\text { J17-3a/b }\end{array}$ & $\begin{array}{c}\text { CMS-1000,a } \\
\text { J17-6a/b }\end{array}$ & $\begin{array}{l}\text { Polytrope }^{c} \\
\text { [WH16] }\end{array}$ & $\begin{array}{l}\text { CMS [HM16] } \\
\text { DFT-MD } 7.13\end{array}$ & $\begin{array}{c}\text { Juno } 0.9^{d} \\
{[\text { CS17] }}\end{array}$ & $\begin{array}{c}\text { CMS-1000 } \\
\mathrm{J} 17-4 \mathrm{z}\end{array}$ \\
\hline$J_{2} / 10^{-6}$ & $14696.514 \pm 0.272$ & 14696.6 & $\begin{array}{l}14698.30^{a} \\
14696.50^{b}\end{array}$ & $\begin{array}{l}14698.24^{a} \\
14696.64^{b}\end{array}$ & 13988.15 & 14696.43 & 14690.68 & 14692.26 \\
\hline$-J_{4} / 10^{-6}$ & $586.623 \pm 0.363$ & 586.64 & 586.65 & $\begin{array}{l}586.62^{a} \\
586.63^{b}\end{array}$ & 531.83 & 596.05 & 581.91 & 582.00 \\
\hline$J_{6} / 10^{-6}$ & $34.244 \pm 0.236$ & 34.09 & 34.21 & 34.42 & 30.12 & 35.15 & 31.75 & 33.85 \\
\hline$-J_{8} / 10^{-6}$ & $2.502 \pm 0.311$ & 2.732 & 2.460 & 2.491 & 2.132 & 2.546 & 1.335 & 2.433 \\
\hline$J_{10} / 10^{-7}$ & $\ldots$ & $\ldots$ & 2.021 & 2.057 & 1.741 & 2.10 & $\ldots$ & 1.999 \\
\hline$-J_{12} / 10^{-8}$ & $\ldots$ & $\ldots$ & 1.821 & 1.860 & 1.568 & $\ldots$ & $\ldots$ & 1.801 \\
\hline$J_{14} / 10^{-9}$ & $\cdots$ & $\ldots$ & 1.755 & 1.797 & 1.518 & $\ldots$ & $\ldots$ & 1.736 \\
\hline$-J_{16} / 10^{-10}$ & $\ldots$ & $\ldots$ & 1.781 & 1.827 & 1.552 & $\ldots$ & $\ldots$ & 1.762 \\
\hline$J_{18} / 10^{-11}$ & $\ldots$ & $\ldots$ & 1.883 & 1.934 & 1.656 & $\ldots$ & $\ldots$ & 1.862 \\
\hline
\end{tabular}

Notes. ${ }^{(a)}$ Fit to $J_{2}$ and $J_{4}$ Juno data using ToF 4 ; ${ }^{(b)}$ same as $(a)$ but for $J_{2}$ value to be fitted shifted by the difference ToF 4 minus CMS, values are displayed only where different from $(a) ;{ }^{(c)}$ Bessel solution for rigid rotation; ${ }^{(d)}$ Juno data corrected for zonal winds based on Bessel solution for the $\Delta J_{2 n}$ of polytropic model with wind depth HAWD $=0.9 ;^{(e)}$ same as $(a)$ but using the $J_{2}$ and $J_{4}$ values from $(d)$.

References. [F17] = Folkner et al. (2017), [WH16] = Wisdom \& Hubbard (2016), [HM16] = Hubbard \& Militzer (2016), [CS17] = Cao \& Stevenson (2017).

depth of $7000 \mathrm{~km}\left(0.9 R_{\mathrm{J}}\right)$. Furthermore, application of the more accurate CMS method in combination with the physical EOS $\mathrm{H} / \mathrm{He}-\mathrm{REOS} .3$ is found to yield higher-order $\left|J_{2 n}\right|$ values that are $10 \%$ higher than the prediction from the polytropic model (Table 1).

Still, the internal structure of Jupiter remains poorly constrained. Further insight might be gained from a Juno measurement of the fluid Love number $k_{2}$ and its consideration in three-dimensional models for the gravity field (Wahl et al. 2017a).

Acknowledgements. I thank Naor Movshovitz for sharing results for linear density models with the CMS method, Ronald Redmer and Ravit Helled for inspiring discussions, and the Referee and the Scientific Editor for helpful comments that improved the paper. This work was supported by the DFG grant NE1734/1-1 of the German Science Foundation.

\section{References}

Becker, A., Lorenzen, W., Fortney, J. J., et al. 2014, ApJS, 215, 14 Cao, H., \& Stevenson, D. 2017, J. Geophys. Res. Planets, 122, 686 Folkner, W., Iess, L., Anderson, J., et al. 2017, Geophys. Res. Lett., 44, 4694 Fortney, J. J., \& Nettelmann, N. 2010, Springer Space Sci. Rev., 152, 423 Helled, R. 2011, ApJ, 735, L16

Helled, R., \& Guillot, T. 2013, ApJ, 767, 113
Helled, R., \& Stevenson, D. 2017, ApJ, 840, L4

Hubbard, W. B. 1999, Icarus, 137, 357

Hubbard, W. B. 2013, ApJ, 768, 43

Hubbard, W. B., \& Militzer, B. 2016, ApJ, 820, 80

Hubbard, W., Schubert, G., Kong, D., \& Zhang, K. 2014, Icarus, 242, 138

Kaspi, Y., Hubbard, W., Showman, A., \& Flierl, G. 2010, Geophys. Res. Lett., 37, L01204

Leconte, J., \& Chabrier, G. 2012, A\&A, 540, A20

Lodders, K. 2003, ApJ, 591, 1220

Mankovich, C., Fortney, J., \& Moore, K. 2016, ApJ, 832, 113

Miguel, Y., Guillot, T., \& Fayon, L. 2016, A\&A, 596, A114

Militzer, B., \& Hubbard, W. B. 2013, ApJ, 774, 148

Militzer, B., Hubbard, W. B., Vorberger, J., Tamblyn, I., \& Bonev, S. B. 2008, ApJ, 688, L54

Nettelmann, N. 2011, Ap\&SS, 336, 47

Nettelmann, N., Becker, A., Holst, B., \& Redmer, R. 2012, ApJ, 750, 52

Nettelmann, N., Fortney, J. J., Moore, K., \& Mankovich, C. 2015, MNRAS, 447, 3422

Saumon, D., \& Guillot, T. 2004, ApJ, 609, 1170

Vazan, A., Helled, R., Podolak, M., \& Kovetz, A. 2016, ApJ, 829, 118

Venturini, J., Alibert, Y., \& Benz, W. 2016, A\&A, 596, A90

Wahl, S., Hubbard, W., \& Militzer, B. 2017a, Icarus, 282, 183

Wahl, S., Hubbard, W., Militzer, B., et al. 2017b, Geophys. Res. Lett., 44, 4649

Wisdom, J. 1996, Non-Perturbative Hydrostatic Equilibrium, http://web.mit. edu/wisdom/interior . pdf

Wisdom, J., \& Hubbard, W. B. 2016, Icarus, 267, 315

Zhang, K., Kong, D., \& Schubert, G. 2015, ApJ, 806, 270

Zharkov, V. N., \& Trubitsyn, V. P. 1978, Physics of Planetary Interiors (Tucson: Parchart) 
Table A.1. CMS results for linear density model.

\begin{tabular}{rccc}
\hline \hline$J_{2 n}$ & $\begin{array}{c}\text { CMS-128 } \\
{[\mathrm{H} 13]}\end{array}$ & $\begin{array}{c}\text { CMS-128 } \\
\text { this }\end{array}$ & $\begin{array}{c}\text { CMS-512 } \\
\text { work }\end{array}$ \\
\hline$J_{2} / 10^{-2}$ & 1.4798138 & 1.47981376 & 1.47978941 \\
$-J_{4} / 10^{-4}$ & 5.9269129 & 5.92691294 & 5.92726570 \\
$J_{6} / 10^{-5}$ & 3.4935680 & 3.49356798 & 3.49433822 \\
$-J_{8} / 10^{-6}$ & 2.5493209 & 2.54932089 & 2.55049835 \\
$J_{10} / 10^{-7}$ & 2.1308951 & 2.13089515 & 2.13255938 \\
$-J_{12} / 10^{-8}$ & 1.9564143 & 1.95641425 & 1.95871536 \\
$J_{14} / 10^{-9}$ & 1.9237724 & 1.92377252 & 1.92693981 \\
\hline
\end{tabular}

Notes. All parameters are chosen as in [H13] $=$ Hubbard (2013).

\section{Appendix A: Linear density models with CMS}

Hubbard (2013) provides the gravitational harmonics for a linear density model with Jupiter-like parameters and $N=128$. I use that model to test my implementation of the CMS method. The agreement is excellent for the given number of digits (seven in H13; see Table A.1). However, a model with only $N=128$ is not yet converged as the same linear density model with $N=512$ shows. Therefore, in order to obtain this good agreement with the linear density model of H13, I had to choose the spacings in equatorial radius $\mathrm{d} \lambda_{i}$ and the dimensionless density jumps $\delta_{i}$ exactly as in H13. In particular (N. Movshovitz, pers. comm.), $\lambda_{0}=1, \lambda_{N}=0, \delta_{0}=\delta_{N}=0, \mathrm{~d} \lambda=1 /(N-1), \lambda_{1}=\lambda_{0}-\mathrm{d} \lambda / 2$, $\lambda_{i}=\lambda_{i-1}-\mathrm{d} \lambda$ for $i>1$, and $\delta_{i}=\mathrm{d} \lambda_{i}$ for $i \geq 1$. All other parameters were chosen as in H13, in particular $q=0.088822426$, $R_{\mathrm{eq}}=71492 \mathrm{~km}$, and GM $=126686536 \mathrm{~km}^{3} / \mathrm{s}^{2}$.

\section{Appendix B: ToF to fourth-order coefficients}

I summarize the theory of figures of Zharkov \& Trubitsyn (1978) and then give the coefficients up to the fourth order. Consider a spheroidal planet in hydrostatic equilibrium of density distribution $\rho(r, \vartheta)$ which is symmetric with respect to the axis of rotation and the equatorial plane. As a result, there is no dependence on azimuthal angle $\varphi$, and only even indices in the spherical harmonics expansions survive. In this two-dimensional problem, a surface of constant total potential $U$ only depends on polar angle $\vartheta$. Different such surfaces $r_{l}(\vartheta)$ are labeled by the level parameter $l$. In the ToF method according to Zharkov \& Trubitsyn (1978), $l$ is taken to be the mean radius of the respective equipotential surface as defined by the condition of equal volume, $(4 \pi / 3) l^{3}=2 \pi \int_{-1}^{1} \mathrm{~d} \cos \vartheta \int_{0}^{r_{l}(\vartheta)} \mathrm{d} r^{\prime} r^{\prime 2}$. Furthermore, any dependence on $(r, \vartheta)$ is replaced by dependence on $(l, \vartheta)$ through the expansion of $r_{l}(\vartheta)$ into a series of Legendre polynomials $P_{n}(\cos \vartheta)$ according to

$r_{l}(\vartheta)=l\left(1+\sum_{n=0}^{\infty} s_{2 n}(l) P_{2 n}(\cos \vartheta)\right)$,

where the $s_{2 n}(l)$ are the figure functions. The first-order deviation from a spherical shape is described by $s_{2}$, while $s_{0}$ can be determined with the help of the equal-volume condition to

$s_{0}=-\frac{1}{5} s_{2}^{2}-\frac{2}{105} s_{2}^{3}-\frac{1}{9} s_{4}^{2}-\frac{2}{35} s_{2}^{2} s_{4}$.

The figure functions $s_{2 n}$ are of $n$th order except $s_{0}$ which is of second order. In the following, I abbreviate the expression in parenthesis in Eq. (B.1) by $(1+\Sigma)$ and set $\mu=\cos \vartheta$.
The total potential is composed of the gravitational potential $V(\boldsymbol{r})=-G \int \mathrm{d}^{3} r^{\prime} \rho /\left|\boldsymbol{r}^{\prime}-\boldsymbol{r}\right|$ while the centrifugal potential reads $Q=-\frac{1}{2} \omega^{2} r^{2} \sin ^{2} \vartheta$. In the ToF it is convenient to capture the centrifugal term due to the planetary rotation of angular rotation rate $\omega$ by the small parameter $m=\omega^{2} R_{\mathrm{m}}^{3} / G M$, where $R_{\mathrm{m}}$ is the mean radius of the outermost level surface. After expanding $V$ and $Q$ into series of Legendre polynomials and replacing $r$ by Eq. (B.1), one can write

$U(l, \vartheta)=-\frac{4 \pi}{3} G \bar{\rho} l^{2} \sum_{k=0}^{\infty} A_{2 k}(l) P_{2 k}(\mu)$,

where $\bar{\rho}$ denotes the mean density $3 M /\left(4 \pi R_{\mathrm{m}}^{3}\right)$. On equipotential surfaces, $\mathrm{d} U / \mathrm{d} \theta=0$ and thus $A_{2 k} \equiv 0$ for $k>0$. This property is used to determine the $s_{2 n}$, while $A_{0}$ yields the total potential. One finds

$$
\begin{aligned}
A_{0}= & \left(1+\frac{2}{5} s_{2}^{2}-\frac{4}{105} s_{2}^{3}+\frac{2}{9} s_{4}^{2}+\frac{43}{175} s_{2}^{4}-\frac{4}{35} s_{2}^{2} s_{4}\right) S_{0} \\
& +\left(-\frac{3}{5} s_{2}+\frac{12}{35} s_{2}^{2}-\frac{234}{175} s_{2}^{3}+\frac{24}{35} s_{2} s_{4}\right) S_{2}+\left(\frac{6}{7} s_{2}^{2}-\frac{5}{9} s_{4}\right) S_{4} \\
& +S_{0}^{\prime}+\left(\frac{2}{5} s_{2}+\frac{2}{35} s_{2}^{2}+\frac{4}{35} s_{2} s_{4}-\frac{2}{25} s_{2}^{3}\right) S_{2}^{\prime}+\left(\frac{4}{9} s_{4}\right. \\
& \left.+\frac{12}{35} s_{2}^{2}\right) S_{4}^{\prime}+\frac{m}{3}\left(1-\frac{2}{5} s_{2}-\frac{9}{35} s_{2}^{2}-\frac{4}{35} s_{2} s_{4}+\frac{22}{525} s_{2}^{3}\right) .
\end{aligned}
$$

The functions $S_{n}$ and $S_{n}^{\prime}$ will be defined below.

\section{B.1. From $V$ to $D_{n}, D_{n}^{\prime}$ and further to $S_{n}, S_{n}^{\prime}$, and $J_{n}$}

The gravitational potential at a location $(r, \vartheta)$ in the planet separates into an external potential $r>r^{\prime}$ due to the mass distribution $\rho\left(r^{\prime}, \vartheta^{\prime}\right)$ interior to $r$ and an internal potential $r<r^{\prime}$ due to the mass distribution $\rho\left(r^{\prime}, \vartheta^{\prime}\right)$ exterior to $r$. The multipole expansion of $V$ reads

$V(r, \vartheta)=-\frac{G}{r} \sum_{n=0}^{\infty}\left(r^{-2 n} D_{2 n}(r)+r^{2 n+1} D_{2 n}^{\prime}(r)\right) P_{2 n}(\mu)$.

Using Eq. (B.1), the volume integrals $D_{n}$ of the external and $D_{n}^{\prime}$ of the internal gravity field expansion take the form ${ }^{2}$

$$
\begin{aligned}
& D_{n}(l)=\frac{2 \pi}{n+3} \int_{0}^{l} \mathrm{~d} l^{\prime} \rho\left(l^{\prime}\right) \int_{-1}^{1} \mathrm{~d} \mu^{\prime} P_{n}\left(\mu^{\prime}\right) \frac{\mathrm{d} r^{n+3}}{\mathrm{~d} l} \\
& D_{n}^{\prime}(l)=\frac{2 \pi}{2-n} \int_{l}^{R_{\mathrm{m}}} \mathrm{d} l^{\prime} \rho\left(l^{\prime}\right) \int_{-1}^{1} \mathrm{~d} \mu^{\prime} P_{n}\left(\mu^{\prime}\right) \frac{\mathrm{d} r^{(2-n)}}{\mathrm{d} l^{\prime}} \quad(n \neq 2) \\
& D_{2}^{\prime}(l)=2 \pi \int_{l}^{R_{\mathrm{m}}} \mathrm{d} l^{\prime} \rho\left(l^{\prime}\right) \int_{-1}^{1} \mathrm{~d} \mu^{\prime} P_{2}\left(\mu^{\prime}\right) \frac{\mathrm{d} \ln r}{\mathrm{~d} l^{\prime}}
\end{aligned}
$$

With $z:=l / R_{\mathrm{m}}$, their dimensionless form is defined as

$S_{n}(z)=\frac{3}{4 \pi \bar{\rho} l^{n+3}} D_{n}(l), \quad S_{n}^{\prime}(z)=\frac{3}{4 \pi \bar{\rho} l^{2-n}} D_{n}^{\prime}(l)$,

2 In the representation by equipotential surfaces, $r<l^{\prime}$ can happen for the external field and $r>l^{\prime}$ for the internal field. This does not pose a problem here (Zharkov \& Trubitsyn 1978) as long as the $m$-value is sufficiently small (Hubbard et al. 2014). 
and can be written as

$$
\begin{aligned}
& S_{n}(z)=\frac{1}{z^{n+3}} \int_{0}^{z} \mathrm{~d} z^{\prime} \frac{\rho\left(z^{\prime}\right)}{\bar{\rho}} \frac{\mathrm{d}}{\mathrm{d} z^{\prime}}\left[z^{n+3} f_{n}\left(z^{\prime}\right)\right] \\
& S_{n}^{\prime}(z)=\frac{1}{z^{(2-n)}} \int_{z}^{1} \mathrm{~d} z^{\prime} \frac{\rho\left(z^{\prime}\right)}{\bar{\rho}} \frac{\mathrm{d}}{\mathrm{d} z^{\prime}}\left[z^{\prime 2-n} f_{n}^{\prime}\left(z^{\prime}\right)\right] \\
& S_{0}(z)=\frac{m(z)}{M z^{3}} .
\end{aligned}
$$

After application of partial integration and assuming $\mathrm{d} \rho / \mathrm{d} z$ to be finite, the $S_{n}, S_{n}^{\prime}$ adopt the convenient form for numerical evaluation

$$
\begin{aligned}
& S_{n}(z)=\frac{\rho(z)}{\bar{\rho}} f_{n}(z)-\frac{1}{z^{n+3}} \int_{0}^{z} \frac{\mathrm{d} \rho}{\bar{\rho}} z^{\prime n+3} f_{n}\left(z^{\prime}\right) \\
& S_{n}^{\prime}(z)=-\frac{\rho(z)}{\bar{\rho}} f_{n}^{\prime}(z)+\frac{1}{z^{2-n}}\left(\frac{\rho(1)}{\bar{\rho}} f_{n}^{\prime}(1)-\int_{z}^{1} \frac{\mathrm{d} \rho}{\bar{\rho}} z^{\prime 2-n} f_{n}^{\prime}\left(z^{\prime}\right)\right)
\end{aligned}
$$

with

$$
\begin{aligned}
f_{n}(z) & =\frac{3}{2(n+3)} \int_{-1}^{1} \mathrm{~d} \mu P_{n}(\mu)(1+\Sigma)^{n+3}, \\
f_{n}^{\prime}(z) & =\frac{3}{2(2-n)} \int_{-1}^{1} \mathrm{~d} \mu P_{n}(\mu)(1+\Sigma)^{2-n} \quad(n \neq 2), \\
f_{2}^{\prime}(z) & =\frac{3}{2} \int_{-1}^{1} \mathrm{~d} \mu P_{n}(\mu) \ln (1+\Sigma) .
\end{aligned}
$$

By expressing powers of $(1+\Sigma)$ in terms of the binomial series expansions, and by further expanding powers of $\Sigma$ into linear series of Legendre polynomials and by making use of $\int_{-1}^{1} \mathrm{~d} \mu P_{n}(\mu) P_{m}(\mu)=0$ for $n \neq m$, the integrals in Eqs. (B.10) can be solved analytically. The results for $f_{n}(z)$ and $f_{n}^{\prime}(z)$ are provided in Eqs. (B.16) and (B.17). Accordingly, the integrals $S_{2 n}$ and $S_{2 n}^{\prime}$ are of $n$th order. Finally, the gravitational harmonics are obtained as

$J_{2 n}=-\left(R_{\mathrm{m}} / R_{\mathrm{eq}}\right)^{2 n} S_{2 n}(1)$

\section{B.2. Coefficients in $A_{2 n}$ for computing the $s_{2 n}$}

Below, I give the coefficients that are of fourth order or lower after multiplication with $m, S_{2 n}$, or $S_{2 n}^{\prime}$ as occurring in the respective equations. They were generated by a $\mathrm{C}++$ program written by myself in 2004 ,

$$
\begin{aligned}
A_{2}= & \left(-s_{2}+\frac{2}{7} s_{2}^{2}+\frac{4}{7} s_{2} s_{4}-\frac{29}{35} s_{2}^{3}+\frac{100}{693} s_{4}^{2}+\frac{454}{1155} s_{2}^{4}\right. \\
& \left.-\frac{36}{77} s_{2}^{2} s_{4}\right) S_{0}+\left(1-\frac{6}{7} s_{2}-\frac{6}{7} s_{4}+\frac{111}{35} s_{2}^{2}-\frac{1242}{385} s_{2}^{3}\right. \\
& \left.+\frac{144}{77} s_{2} s_{4}\right) S_{2}+\left(-\frac{10}{7} s_{2}-\frac{500}{693} s_{4}+\frac{180}{77} s_{2}^{2}\right) S_{4} \\
& +\left(1+\frac{4}{7} s_{2}+\frac{1}{35} s_{2}^{2}+\frac{4}{7} s_{4}-\frac{16}{105} s_{2}^{3}+\frac{24}{77} s_{2} s_{4}\right) S_{2}^{\prime} \\
& +\left(\frac{8}{7} s_{2}+\frac{72}{77} s_{2}^{2}+\frac{400}{693} s_{4}\right) S_{4}^{\prime} \\
& +\frac{m}{3}\left(-1+\frac{10}{7} s_{2}+\frac{9}{35} s_{2}^{2}-\frac{4}{7} s_{4}+\frac{20}{77} s_{2} s_{4}-\frac{26}{105} s_{2}^{3}\right) .
\end{aligned}
$$

To lowest order, $s_{2} \approx-m / 3$, thus $s_{2}$ is of first order in $m$,

$$
\begin{aligned}
A_{4}= & \left(-s_{4}+\frac{18}{35} s_{2}^{2}-\frac{108}{385} s_{2}^{3}+\frac{40}{77} s_{2} s_{4}+\frac{90}{143} s_{2} s_{6}+\frac{162}{1001} s_{4}^{2}\right. \\
& \left.+\frac{16902}{25025} s_{2}^{4}-\frac{7369}{5005} s_{2}^{2} s_{4}\right) s_{0}+\left(-\frac{54}{35} s_{2}-\frac{60}{77} s_{4}+\frac{648}{385} s_{2}^{2}\right. \\
& \left.-\frac{135}{143} s_{6}+\frac{21468}{5005} s_{2} s_{4}-\frac{122688}{25025} s_{2}^{3}\right) S_{2}+\left(1-\frac{100}{77} s_{2}\right. \\
& \left.-\frac{810}{1001} s_{4}+\frac{6368}{1001} s_{2}^{2}\right) S_{4}-\frac{315}{143} s_{2} S_{6}+\left(\frac{36}{35} s_{2}\right. \\
& \left.+\frac{108}{385} s_{2}^{2}+\frac{40}{77} s_{4}+\frac{3578}{5005} s_{2} s_{4}-\frac{36}{175} s_{2}^{3}+\frac{90}{143} s_{6}\right) S_{2}^{\prime} \\
& +\left(1+\frac{80}{77} s_{2}+\frac{1346}{1001} s_{2}^{2}+\frac{648}{1001} s_{4}\right) S_{4}^{\prime}+\frac{270}{143} s_{2} S_{6}^{\prime} \\
& +\frac{m}{3}\left(-\frac{36}{35} s_{2}+\frac{114}{77} s_{4}+\frac{18}{77} s_{2}^{2}-\frac{978}{5005} s_{2} s_{4}+\frac{36}{175} s_{2}^{3}-\frac{90}{143} s_{6}\right) .
\end{aligned}
$$

To lowest order, $s_{4} \sim m \times s_{2}$, thus $s_{4}$ is of second order in $m$,

$$
\begin{aligned}
A_{6}= & \left(-s_{6}+\frac{10}{11} s_{2} s_{4}-\frac{18}{77} s_{2}^{3}+\frac{28}{55} s_{2} s_{6}+\frac{72}{385} s_{2}^{4}+\frac{20}{99} s_{4}^{2}\right. \\
& \left.-\frac{54}{77} s_{2}^{2} s_{4}\right) S_{0}+\left(-\frac{15}{11} s_{4}+\frac{108}{77} s_{2}^{2}-\frac{42}{55} s_{6}-\frac{144}{77} s_{2}^{3}\right. \\
& \left.+\frac{216}{77} s_{2} s_{4}\right) S_{2}+\left(-\frac{25}{11} s_{2}-\frac{100}{99} s_{4}+\frac{270}{77} s_{2}^{2}\right) S_{4} \\
& +\left(1-\frac{98}{55} s_{2}\right) S_{6}+\left(\frac{10}{11} s_{4}+\frac{18}{77} s_{2}^{2}+\frac{36}{77} s_{2} s_{4}+\frac{28}{55} s_{6}\right) S_{2}^{\prime} \\
& +\left(\frac{20}{11} s_{2}+\frac{108}{77} s_{2}^{2}+\frac{80}{99} s_{4}\right) S_{4}^{\prime}+\left(1+\frac{84}{55} s_{2}\right) S_{6}^{\prime} \\
& +\frac{m}{3}\left(-\frac{10}{11} s_{4}-\frac{18}{77} s_{2}^{2}+\frac{34}{77} s_{2} s_{4}+\frac{82}{55} s_{6}\right) .
\end{aligned}
$$

To lowest order, $s_{6} \sim m \times s_{4}$, thus $s_{6}$ is of third order in $m$.

$$
\begin{aligned}
A_{8}= & \left(-s_{8}+\frac{56}{65} s_{2} s_{6}+\frac{72}{715} s_{2}^{4}+\frac{490}{1287} s_{4}^{2}-\frac{84}{143} s_{2}^{2} s_{4}\right) S_{0} \\
& +\left(-\frac{84}{65} s_{6}-\frac{144}{143} s_{2}^{3}+\frac{336}{143} s_{2} s_{4}\right) S_{2}+\left(-\frac{2450}{1287} s_{4}\right. \\
& \left.+\frac{420}{143} s_{2}^{2}\right) S_{4}-\frac{196}{65} s_{2} S_{6}+S_{8}+\left(\frac{56}{65} s_{6}+\frac{56}{143} s_{2} s_{4}\right) S_{2}^{\prime} \\
& +\left(\frac{1960}{1287} s_{4}+\frac{168}{143} s_{2}^{2}\right) S_{4}^{\prime}+\frac{168}{65} s_{2} S_{6}^{\prime}+S_{8}^{\prime} \\
& +\frac{m}{3}\left(-\frac{56}{65} s_{6}-\frac{56}{143} s_{2} s_{4}\right) .
\end{aligned}
$$

To lowest order, $s_{2 n} \sim m^{n}$.

\section{B.3. Coefficients for computing the $f_{2 n}$ and $f^{\prime}{ }_{2 n}$}

$$
\begin{aligned}
f_{0}= & 1 \\
f_{2}= & \frac{3}{5} s_{2}+\frac{12}{35} s_{2}^{2}+\frac{6}{175} s_{2}^{3}+\frac{24}{35} s_{2} s_{4}+\frac{40}{231} s_{4}^{2}+\frac{216}{385} s_{2}^{2} s_{4} \\
& -\frac{184}{1925} s_{2}^{4}
\end{aligned}
$$




$$
\begin{aligned}
f_{4}= & \frac{1}{3} s_{4}+\frac{18}{35} s_{2}^{2}+\frac{40}{77} s_{2} s_{4}+\frac{36}{77} s_{2}^{3}+\frac{90}{143} s_{2} s_{6}+\frac{162}{1001} s_{4}^{2} \\
& +\frac{6943}{5005} s_{2}^{2} s_{4}+\frac{486}{5005} s_{2}^{4} \\
f_{6}= & \frac{3}{13} s_{6}+\frac{120}{143} s_{2} s_{4}+\frac{72}{143} s_{2}^{3}+\frac{336}{715} s_{2} s_{6}+\frac{80}{429} s_{4}^{2} \\
& +\frac{216}{143} s_{2}^{2} s_{4}+\frac{432}{715} s_{2}^{4} \\
f_{8}= & \frac{3}{17} s_{8}+\frac{168}{221} s_{2} s_{6}+\frac{2450}{7293} s_{4}^{2}+\frac{3780}{2431} s_{2}^{2} s_{4}+\frac{1296}{2431} s_{2}^{4} \\
f_{0}^{\prime}= & \frac{3}{2}-\frac{3}{10} s_{2}^{2}-\frac{2}{35} s_{2}^{3}-\frac{1}{6} s_{4}^{2}-\frac{6}{35} s_{2}^{2} s_{4}+\frac{3}{50} s_{2}^{4} \\
f_{2}^{\prime}= & \frac{3}{5} s_{2}-\frac{3}{35} s_{2}^{2}-\frac{6}{35} s_{2} s_{4}+\frac{36}{175} s_{2}^{3}-\frac{10}{231} s_{4}^{2}-\frac{17}{275} s_{2}^{4} \\
& +\frac{36}{385} s_{2}^{2} s_{4} \\
f_{4}^{\prime}= & \frac{1}{3} s_{4}-\frac{9}{35} s_{2}^{2}-\frac{20}{77} s_{2} s_{4}-\frac{45}{143} s_{2} s_{6}-\frac{81}{1001} s_{4}^{2}+\frac{1}{5} s_{2}^{2} s_{4} \\
= &
\end{aligned}
$$

$$
\begin{aligned}
f_{6}^{\prime}= & \frac{3}{13} s_{6}-\frac{75}{143} s_{2} s_{4}+\frac{270}{1001} s_{2}^{3}-\frac{50}{429} s_{4}^{2}+\frac{810}{1001} s_{2}^{2} s_{4} \\
& -\frac{54}{143} s_{2}^{4}-\frac{42}{143} s_{2} s_{6} \\
f_{8}^{\prime}= & \frac{3}{17} s_{8}-\frac{588}{1105} s_{2} s_{6}-\frac{1715}{7293} s_{4}^{2}+\frac{2352}{2431} s_{2}^{2} s_{4}-\frac{4536}{12155} s_{2}^{4} .
\end{aligned}
$$

Numerical values for the $s_{2 n}$ and $J_{2 n}$ for a given barotrope $\rho(P)$ can be obtained through an iterative procedure. For given values of the $J_{2 n}$ and $s_{2 n}$, which can initially be zero, the density distribution $\rho(l)$ is computed by numerical integration of the hydrostatic balance equation $\mathrm{d} P / \mathrm{d} l=-\rho(P) \mathrm{d} U / \mathrm{d} l$ using Eq. (B.4), and by integration of the mass conservation equation $\mathrm{d} m / \mathrm{d} l=4 \pi l^{2} \rho(l)$. Given then $\rho(l)$, new figure functions are repeatedly calculated until convergence using Eqs. (B.12) to (B.15), and then the $J_{2 n}$ are calculated using Eq. (B.11). Converged $J_{2 n}$ values for a given barotrope require about six iterations of this procedure. 